\title{
Identification of transcripts overexpressed during airway epithelium differentiation
}

\author{
B. Chhin ${ }^{\star, \#, f}$, J.T. Pham*,\#,f , L. El Zein ${ }^{\star, \#}$, K. Kaiser ${ }^{\star}$, O. Merrot ${ }^{+}$and P. Bouvagnet ${ }^{\star \star \#, \S}$
}

ABSTRACT: Human airway epithelium, the defence at the forefront of protecting the respiratory tract, evacuates inhaled particles by a permanent beating of epithelial cell cilia. When deficient, this organelle causes primary ciliary dyskinesia, and, despite numerous studies, data regarding ciliated cell gene expression remain incomplete. The aim of the present study was to identify genes specifically expressed in human ciliated respiratory cells via transcriptional analysis.

The transcriptome of dedifferentiated epithelial cells was subtracted from that of fully redifferentiated cells using complementary DNA representational difference analysis. In order to validate the results, gene overexpression in ciliated cells was confirmed by real-time PCR, and by comparing the present list of genes overexpressed in ciliated cells to lists obtained in previous studies.

A total of 53 known and 12 unknown genes overexpressed in ciliated cells were identified. The majority $(66 \%)$ of known genes had never previously been reported as being involved in ciliogenesis, and the unknown genes represent hypothetical novel transcript isoforms or new genes not yet reported in databases. Finally, several genes identified here were located in genomic regions involved in primary ciliary dyskinesia by linkage analysis.

In conclusion, the present study revealed sequences of new cilia-related genes, new transcript isoforms and novel genes which should be further characterised to aid understanding of their function(s) and their probable disorder-related involvement.

KEYWORDS: Airway epithelium, cilia, human, representational difference analysis, transcriptome

$\mathbf{T}$ he airway epithelium is a pseudo-stratified layer, consisting of specialised cell types, including basal cells, goblet/secretory cells and ciliated columnar cells. It plays a critical role in airway defence by protecting the respiratory tract from infections and damage induced by inhaled toxins, pathogens and particles. It constitutes a physical barrier against environmental aggression, through secreted factors that mediate the host immune system and through mucociliary clearance. On respiratory cells, ciliary beat defects cause a disease referred to as primary ciliary dyskinesia (PCD). Cilia are hair-like organelles which can be present on respiratory cells and on many other human cells. Cilia of all types exhibit numerous similarities, but they differ depending on their motility or sensory function. An increasing interest in respiratory epithelia has led researchers to elucidate genes acting in ciliogenesis.

Proteomic analyses have been used to identify components located in the axonemes or centrioles of cilia in humans or flagella in other well-known organisms [1-5]. Comparative genomic searches led to the detection of genes conserved in the genome of ciliated organisms versus nonciliated organisms [6, 7]. In order to reveal genes specifically expressed during flagellar regeneration or ciliogenesis, several studies have been carried out using various transcriptional strategies [8-12].

Mutations in several genes revealed by these studies turned out to cause diverse human ciliary diseases, such as polycystic kidney disease, retinal dystrophy, neurosensory impairment, Bardet-Biedl syndrome, oral-facial-digital syndrome type 1 and PCD, demonstrating that these genes should be considered in deciphering the aetiology of ciliopathies [13]

Using a different approach, to discover genes specifically expressed in human ciliated respiratory cells that could be responsible for human disorders, a method referred to as representational difference analysis (RDA) was used. This method, first described by LISITSYN et al. [14], represents a process of subtraction coupled to amplification and was initially applied to the detection of differences between two genomes. Subsequently, HuBANK and ScHATZ [15] adapted RDA for use with complementary DNA (cDNA)
AFFILIATIONS

*Laboratory of Cardiogenetics, Laboratory Team 4171, and

"Parasitology, University of Lyon 1 , \#Laboratory of Cardiogenetics, Methodology Research Team 0107 National Institute for Health and Medical Research,

${ }^{+}$Ear, Nose and Throat Service, CroixRousse Hospital, Hospices Civils de Lyon,

${ }^{\S}$ Laboratory of Cardiogenetics, East Centre of Biology and Pathology, Groupe Hospitalier Est, Hospices Civils de Lyon, Lyon, France.

${ }^{f}$ Both authors contributed equally to this study.

CORRESPONDENCE

P. Bouvagnet, Laboratoire

Cardiogénétique, CBPE, Groupe Hospitalier Est, 59 boulevard Pinel, 69677 Bron, France. Fax: 33427855900

E-mail: Patrice.Bouvagnet@ recherche.univ-lyon1.fr

Received:

December 192007

Accepted after revision:

February 082008

SUPPORT STATEMENT

B. Chhin was supported by a stipend from the French Association against Myopathies (Evry, France) between 2004 and 2007. The Laboratory of Cardiogenetics (Hospices Civils de Lyon, Lyon, France) is supported by grants from Scientific Interest Group Rare Diseases 2003 and Hospital Project of Clinical Research regional 2003 (Paris, France), and the Laboratory of Cardiogenetics (University of Lyon 1, Lyon, France) by grants from the ProKartagener Foundation 2005 (Geneva, Switzerland) and Renaud Febvre Foundation (Les Etards, France).

STATEMENT OF INTEREST None declared.

European Respiratory Journal Print ISSN 0903-1936 Online ISSN 1399-3003 
in order to isolate genes expressed differentially in two cell populations.

In the present study, the sequential culture system described by JORISSEN et al. [16], in which epithelial cells covering the turbinates of the nasal cavity are dediffentiated in flat nonciliated cells and then redifferentiated in ciliated cells, was utilised. The transcriptome of flat nonciliated cells was subtracted from that of re-ciliated cells in order to characterise transcripts specific to ciliated cells. Differentially expressed genes were cloned and sequenced, resulting in the identification of bona fide and predicted genes. Moreover, genomic fragments that lay in intergenic intervals were cloned, suggesting the existence of new putative genes. The increased expression of some known and predicted genes during ciliogenesis was confirmed by real-time PCR validation studies.

\section{MATERIALS AND METHODS}

\section{Cell culture}

Human respiratory cells from normal subjects were obtained from nasal turbinates, which were removed and discarded, thereby providing access to the ethmoidal sinus (at the Ear Nose and Throat Service, Croix-Rousse Hospital, Lyon, France). Patients were operated on for tumours located in the ethmoidal region and showed no respiratory disease. Cells were grown using the immerged cell culture method described by JORISSEN et al. [16]. Briefly, ciliated cells were isolated by pronase digestion and expanded in collagen-coated $25-\mathrm{cm}^{2}$ flasks to dedifferentiate in nonciliated cells at $37^{\circ} \mathrm{C}$ under $5 \% \quad \mathrm{CO}_{2}$. When they reached $80-90 \%$ confluence, collagen was digested and cells were suspended in flasks with rotation $\left(80\right.$ revolutions $\left.\cdot \mathrm{min}^{-1}\right)$ at $37^{\circ} \mathrm{C}$ to redifferentiate in the form of ciliated vesicles. Nonciliated cells were collected at $80-90 \%$ confluence, when they stopped proliferating, and vesicles were collected when they were fully covered by cilia.

\section{Isolation of mRNA and complementary DNA synthesis}

RNA was extracted from nonciliated and ciliated cells using Extract-All ${ }_{\circledR}$ (Eurobio, Courtaboeuf, France), following the manufacturer's instructions. $\operatorname{Poly}(\mathrm{A})^{+}$mRNA was separated from total RNA using the Dynabeads Oligo $(\mathrm{dT})_{25}$ purification kit (Dynal Biotech, Oslo, Norway) and its quality was assessed on an agarose gel. cDNA was synthesised from $2.85 \mu \mathrm{g}$ poly(A) mRNA by oligo-deoxythymidine (dT) priming using SuperScript II Reverse Transcriptase as recommended by the manufacturer (Invitrogen, Grand Island, NY, USA). Doublestranded cDNA was prepared in an $80-\mu \mathrm{L}$ total volume, containing $20 \mu \mathrm{L}$ cDNA template, $400 \mu \mathrm{M}$ deoxyribonucleoside triphosphates, 5 U DNA ligase (New England Biolabs, Ipswich, MA, USA), 24 U DNA polymerase (Invitrogen) and $1 \mathrm{U}$ RNase $\mathrm{H}$ (Invitrogen). The reaction was performed for $2 \mathrm{~h}$ at $16^{\circ} \mathrm{C}$ and then supplemented by $6 \mathrm{U}$ T4 DNA polymerase (Invitrogen) for an additional 30-min incubation.

The absence of genomic DNA contamination was confirmed by PCR using $\alpha$-tubulin primers, which could amplify either a 320-bp cDNA fragment or a 468-bp genomic DNA fragment (protocol available on request).

\section{Generation of difference products}

cDNA RDA was performed on the basis of the protocol described by HUBANK and SCHATZ [15] with slight modifications. Double-stranded cDNA $(2 \mu \mathrm{g})$ from the two cell populations was digested with DpnII (New England Biolabs) to generate tester (ciliated cells) and driver (nonciliated cells) cDNA representations. In order to facilitate purification of the digested representations, primers pair sets were biotinylated and removed using a Streptavidin M-280 kit (Dynal Biotech), following the manufacturer's recommendations. The first subtractive hybridisation tester:driver cDNA ratio was 1:50. In the second and third rounds of subtractive hybridisation, the ratio was increased to 1:500 and 1:250,000, and mung bean nuclease digestion of PCR products was omitted (detailed protocol available on request).

\section{Cloning and DNA sequencing}

The products of the third round of the PCR were digested with DpnII, and, to facilitate their identification, bands of 200, 300, 400 and 600 bp were separately gel-purified using a QIAquick gel extraction kit (Qiagen, Germantown, MD, USA). Purified products were shotgun cloned into a BamHI-digested dephosphorylated pBlueScript ${ }$ II KS+ vector (Stratagene, San Diego, CA, USA) and used to transform DH5 $\alpha$ One Shot competent cells (Invitrogen), according to the manufacturer's protocol. Bacteria were plated on Luria-Bertani medium/ampicillin plates and colonies screened for inserts by SacII and XhoI double digestion, following conventional plasmid extraction.

Cloned products were sequenced using the M13-20 primer. The sequencing reaction was set up using the plasmid asa template and the Big Dye ${ }^{\circledR}$ Terminator v1.1 cycle sequencing kit (Applied Biosystems, Foster City, CA, USA) following the manufacturer's instructions. Sequence analysis was performed on a 3100 automated ABI sequencing apparatus (Applied Biosystems), and sequences were aligned using Staden 1.7.0 for Windows from the Staden Package [17] after extraction of primer and vector sequences.

\section{Sequence analysis}

Sequences were formatted using the FAST-All program [18] and compared to the public human genomic databases, Ensembl [19] and National Center for Biotechnology Information [20], with the nucleotide-nucleotide basic local alignment search tool (BLASTN).

\section{Real-time PCR}

Nonciliated and ciliated cells were collected, centrifuged to remove cell medium and washed in PBS ( $\mathrm{pH}$ 7.4). The cell pellet was stored at $-80^{\circ} \mathrm{C}$ until processing. Purified mRNA was prepared using the Chemagic mRNA direct kit (Chemagen, Baesweiler, Germany) following the manufacturer's recommendations. DNA contamination was removed with a DNase I treatment (Invitrogen), and mRNA was quantified using an ND-1000 spectrophometer (NanoDrop, Wilmington, DE, USA). mRNA (10 $\mu \mathrm{g})$ was reverse transcribed to generate cDNA using the Transcriptor First Strand cDNA synthesis kit (Roche Applied Science, Rotkreuz, Switzerland) and anchored oligo $(\mathrm{dT})_{18}$, according to the manufacturer's recommendations. 
TABLE 1 Genes overexpressed in human ciliated respiratory cells: complementary DNA fragments mapping to exonic regions

\begin{tabular}{|c|c|c|c|c|}
\hline NCBI gene ID & Gene symbol & Description & Location & Maximum identity $\#$ \\
\hline \multicolumn{5}{|l|}{ Translation } \\
\hline 6175 & RPLPO & Ribosomal protein, large, P0 & $12 q 24.2$ & 99 \\
\hline 6133 & RPL9 & Ribosomal protein L9 & $4 \mathrm{p} 13$ & 100 \\
\hline 6136 & RPL12 & Ribosomal protein L12 & $9 q 34$ & 97 \\
\hline 9045 & RPL14 & Ribosomal protein L14 & $3 p 22-p 21.2$ & 91 \\
\hline 6228 & RPS23 & Ribosomal protein S23 & $5 q 14.2$ & 100 \\
\hline 6156 & RPL30 & Ribosomal protein L30 & 8q22 & 99 \\
\hline 6161 & RPL32 & Ribosomal protein L32 & 3p25-p24 & 99 \\
\hline 6164 & RPL34 & Ribosomal protein L34 & $4 \mathrm{q} 25$ & 100 \\
\hline 3921 & RPSA & Ribosomal protein SA & 3р22.2 & 97 \\
\hline 10209 & EIF1 & Eukaryotic translation initiation factor 1 & $17 q 21.2$ & 98 \\
\hline \multicolumn{5}{|c|}{$\begin{array}{l}\text { Immunity, inflammation and } \\
\text { defence }\end{array}$} \\
\hline 301 & ANXA1 & Annexin A1 & $9 q 12-q 21.2$ & 100 \\
\hline 967 & CD63 & CD63 molecule & $12 q 12-q 13$ & 99 \\
\hline 284340 & CXCL17 & Chemokine (C-X-C motif) ligand 17 & $19 q 13.2$ & 100 \\
\hline 2280 & FKBP1A & FK506 binding protein $1 \mathrm{~A}, 12 \mathrm{kDa}$ & $20 \mathrm{p} 13$ & 100 \\
\hline 7356 & SCGB1A1 & Secretoglobin, family $1 A$, member 1 (uteroglobin) & $11 q 12.3-q 13.1$ & 99 \\
\hline 5055 & SERPINB2 & Serpin peptidase inhibitor, clade B (ovalbumin), member 2 & $18 q 21.3$ & 100 \\
\hline 7114 & TMSB4X & Thymosin, beta $4, X$-linked & Xq21.3-q22 & 95 \\
\hline 7311 & UBA52 & Ubiquitin A-52 residue ribosomal protein fusion product 1 & $19 p 13.1-p 12$ & 98 \\
\hline \multicolumn{5}{|c|}{ 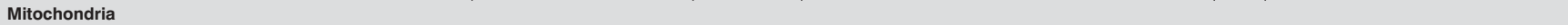 } \\
\hline 514 & ATP5E & ATP synthase, $\mathrm{H}^{+}$transporting, mitochondrial $\mathrm{F} 1$ complex, epsilon subunit & $20 q 13.32$ & $92-97$ \\
\hline 10476 & ATP5H & ATP synthase, $\mathrm{H}^{+}$transporting, mitochondrial F0 complex, subunit d & $17 q 25$ & 99 \\
\hline 539 & ATP5O & ATP synthase, $\mathrm{H}^{+}$transporting, mitochondrial $\mathrm{F} 1$ complex, $\mathrm{O}$ subunit & $21 \mathrm{q} 22.11$ & 97 \\
\hline 4519 & MT-CYB & Mitochondrially encoded cytochrome b & $\mathrm{mt}$ & 100 \\
\hline 4697 & NDUFA4 & $\mathrm{NADH}$ dehydrogenase (ubiquinone) 1 alpha subcomplex, 4, $9 \mathrm{kDa}$ & $7 \mathrm{p} 21.3$ & 99 \\
\hline 58472 & SQRDL & Sulphide quinone reductase-like (yeast) & $15 q 15$ & 97 \\
\hline 29796 & UCRC & Ubiquinol-cytochrome c reductase complex $7.2 \mathrm{kDa}$ & 22cen-q12.3 & 95 \\
\hline \multicolumn{5}{|c|}{$\begin{array}{l}\text { Channels, transporters and } \\
\text { related proteins }\end{array}$} \\
\hline 1173 & AP2M1 & Adaptor-related protein complex 2 , mu 1 subunit & $3 q 28$ & 100 \\
\hline 56888 & KCMF1 & Potassium channel modulatory factor 1 & $2 \mathrm{p} 11.2$ & 99 \\
\hline 26266 & SLC13A4 & Solute carrier family 13 (sodium/sulphate symporters), member 4 & $7 q 33$ & 100 \\
\hline 11254 & SLC6A14 & Solute carrier family 6 (amino acid transporter), member 14 & Xq23-q24 & 98 \\
\hline \multicolumn{5}{|c|}{$\begin{array}{l}\text { Cell signalling and signal } \\
\text { transduction }\end{array}$} \\
\hline 54541 & DDIT4 & DNA-damage-inducible transcript 4 & 10pter-q26.12 & 96 \\
\hline 4092 & SMAD7 & SMAD family member 7 & $18 \mathrm{q} 21.1$ & 97 \\
\hline 221178 & SPATA13 & Spermatogenesis associated 13 & $13 q 12.12$ & 98 \\
\hline 7009 & TEGT & Testis enhanced gene transcript (Bax inhibitor 1) & $12 q 12-q 13$ & 100 \\
\hline \multicolumn{5}{|l|}{ Cytoskeleton } \\
\hline 71 & ACTG1 & Actin, gamma 1 & $17 q 25$ & 98 \\
\hline 3861 & KRT14 & Keratin 14 (epidermolysis bullosa simplex, Dowling-Meara, Koebner) & $17 q 12-q 21$ & 100 \\
\hline $3853 / 140446$ & KRT6A/KRT6C & Keratin $6 \mathrm{~A} / 6 \mathrm{C}$ & $12 q 12-q 13$ & 98 \\
\hline 3855 & KRT7 & Keratin 7 & $12 q 12-q 13$ & $97-100$ \\
\hline 10529 & NEBL & Nebulette & $10 \mathrm{p} 12$ & 100 \\
\hline 7168 & TPM1 & Tropomyosin 1 (alpha) & $15 q 22.1$ & 97 \\
\hline 7170 & TPM3 & Tropomyosin 3 & $1 \mathrm{q} 21.2$ & 94 \\
\hline \multicolumn{5}{|c|}{ Cell proliferation } \\
\hline 996 & CDC27 & Cell division cycle 27 homolog (S. cerevisiae) & $17 q 12-17 q 23.2$ & 94 \\
\hline 10969 & EBNA1BP2 & EBNA1 binding protein 2 & 1 p35-p33 & 98 \\
\hline 3397 & ID1 & Inhibitor of DNA binding 1, dominant negative helix-loop-helix protein & $20 q 11$ & 100 \\
\hline 4831 & NME2 & Non-metastatic cells 2, protein (NM23B) expressed in & $17 q 21.3$ & 97 \\
\hline 57804 & POLD 4 & Polymerase (DNA-directed), delta 4 & $11 \mathrm{q} 13$ & 99 \\
\hline 90441 & ZNF622 & Zinc finger protein 622 & $5 p 15.1$ & 95 \\
\hline \multicolumn{5}{|l|}{ Other } \\
\hline 78996 & C7orf49 & Chromosome 7 open reading frame 49 & $7 \mathrm{q} 33$ & 98 \\
\hline 51637 & C14orf166 & Chromosome 14 open reading frame 166 & $14 q 22.1$ & 99 \\
\hline 26234 & FBXL5 & F-box and leucine-rich repeat protein 5 & $4 p 15.33$ & 100 \\
\hline 10457 & GPNMB & Glycoprotein (transmembrane) nmb & $7 \mathrm{p} 15$ & 96 \\
\hline 51108 & METTL9 & Methyltransferase like 9 & 16p13-p12 & 98 \\
\hline 203068 & TUBB & Tubulin, beta & $6 \mathrm{p} 21.33$ & 98 \\
\hline 7776 & ZNF236 & Zinc finger protein 236 & $18 q 22-q 23$ & 98 \\
\hline
\end{tabular}

NCBI: National Center for Biotechnology Information [20]; mt: mitochondrial; S. cerevisiae: Saccharomyces cerevisiae. ${ }^{\#}$ : between insert and genomic sequence.

Real-time PCR was carried out in a LightCycler System ${ }^{\circledR}$ using the FastStart DNA Master SYBR Green I kit (Roche Applied Science). Reference and target gene primers were obtained from QuantiTect Primer Assays (Qiagen), which contained validated primers sets for the reduced glyceraldehyde-3phosphate dehydrogenase $(G A P D H)$, axonemal dynein intermediate chain 1 (DNAI1), glycoprotein $\mathrm{nmb}(G P N M B)$, retinitis pigmentosa GTPase regulator (RPGR), chromosome 7 open reading frame 49 (C7orf49) and chromosome 14 open reading frame 166 (C14orf166) genes. PCR reactions were set up in a total volume of $20 \mu \mathrm{L}$, containing $2 \mu \mathrm{L}$ SYBR Green FastStart reaction mix, $2.4 \mathrm{mM} \mathrm{MgCl}_{2}, 2 \mu \mathrm{L} 10 \times$ primers mix and $2 \mu \mathrm{L}$
cDNA. The temperature cycling profiles were as follows: $95^{\circ} \mathrm{C}$ for $10 \mathrm{~min}, 40$ cycles of denaturation at $95^{\circ} \mathrm{C}$ for $10 \mathrm{~s}$, annealing at $55^{\circ} \mathrm{C}$ for $10 \mathrm{~s}$, and extension at $72^{\circ} \mathrm{C}$ for $20 \mathrm{~s}$. Melting curve analysis was carried out in the range $65-95^{\circ} \mathrm{C}$ to confirm the specificity of the PCR products.

Gene expression level was determined with the comparative threshold method, using the level of the housekeeping gene GAPDH as a reference value [21]. The threshold cycle of PCR at which amplified product was first detected $(\mathrm{Ct})$ was determined for the real-time PCR. The corrected $\mathrm{Ct}(\Delta \mathrm{Ct})$ for each determination was then used to calculate the relative $n$-fold 
TABLE 2 Genes overexpressed in human ciliated respiratory cells: complentary DNA fragments mapping to intronic regions

\begin{tabular}{|c|c|c|c|c|}
\hline NCBI gene ID & Gene symbol & Description & Location & Maximum identity ${ }^{\#} \%$ \\
\hline 54875 & C9orf39 & Chromosome 9 open reading frame 39 & 9p22.2 & 98 \\
\hline 23464 & GCAT & Glycine C-acetyltransferase (2-amino-3-ketobutyrate coenzyme A ligase) & $22 q 13.1$ & 98 \\
\hline 4862 & NPAS2 & Neuronal PAS domain protein 2 & $2 q 11.2$ & 98 \\
\hline 10196 & РRMT3 & Protein arginine methyltransferase 3 & $11 \mathrm{p} 15.1$ & 99 \\
\hline
\end{tabular}

NCBI: National Center for Biotechnology Information [20]. ${ }^{*}$ : between insert and genomic sequence; ${ }^{\bullet}$ : mapped to two genes (FAM82B and CPNE3); ${ }^{+}$: at the exon-intron junction.

differential expression of a specific gene in a ciliated cell compared with a nonciliated cell sample and expressed as the ratio of the $2^{-\Delta \mathrm{Ct}}$ values.

\section{Statistical analysis}

Data from triplicate experiments are presented as mean \pm SD. For each target gene, the $2^{-\Delta \mathrm{Ct}}$ values of ciliated and nonciliated cells were analysed using an unpaired t-test with the significance set at a $\mathrm{p}$-value of $<0.05$ for a one-tailed test.

\section{RESULTS}

The goal of the present study was to identify genes specifically expressed in human ciliated respiratory cells. To this end, the transcriptome of dedifferentiated epithelial cells was subtracted from that of fully redifferentiated cells. The cDNA RDA procedure used in the present study was closely based on the protocol described by HUBANK and SCHATZ [15], which permits the enrichment of transcripts specifically expressed in a cell type through iterative cycles of amplification/subtraction.

\section{Determination of differentially expressed genes}

Of the bacterial clones, $25 \%$ contained an insert. All clones with an insert $(n=78)$ were sequenced, including four chimeric clones that each contained two cDNA fragments. Altogether, 82 individual cDNA fragments were identified. Four of these
cDNA fragments were recovered several times, and corresponded to the following genes: GPNMB (nine clones), zinc finger protein 236 (ZNF236; six clones), ribosomal protein large P0 (RPLP0; four clones) and ribosomal protein L14 (RPL14; two clones). Finally, 65 unique cDNA fragments were identified and mapped to the genomic human sequence by BLASTN. Of these, $53(82 \%)$ cDNA fragments mapped to exonic sequences of known genes, seven (11\%) to intronic regions or the boundary of an intron-exon junction and five $(7 \%)$ to intergenic regions.

A list of the 53 cDNA fragments corresponding to exonic regions is presented in table 1 . Genes are clustered by their described functions. It is notable that nine ribosomal proteins and an initiation factor involved in translation were identified. Genes related to immunity, inflammation and defence were also detected. The mitochondrial cluster includes seven genes encoding mitochondrial components. Among these, six are nuclear genes and one is mitochondrial (the mitochondrially encoded cytochrome b gene (MT-CYB)). Components of channels, transporters or related proteins are listed in one group, which includes two solute carrier family genes. Genes for cell signalling and signal transduction, such as those encoding spermatogenesis associated 13 (SPATA13) and testis enhanced gene transcript (TEGT; Bax inhibitor 1), are present.

\section{TABLE 3 Complementary DNA fragments mapping to intergenic regions}

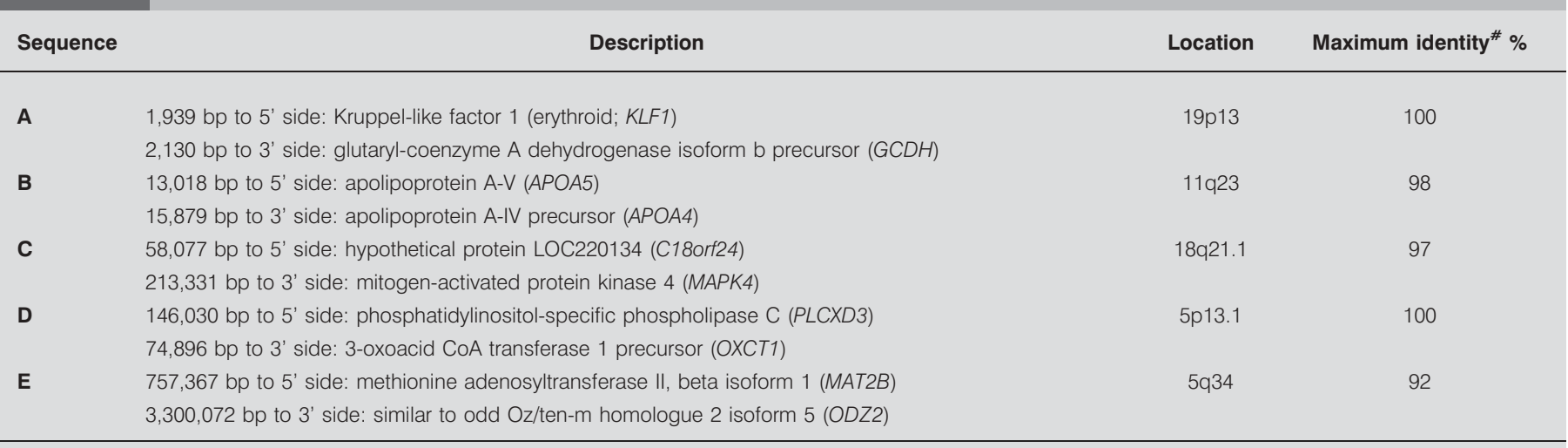

\#: between insert and genomic sequence 


\begin{tabular}{lc} 
TABLE $4 \begin{array}{l}\text { Relative changes in expression of selected } \\
\text { genes }\end{array}$ & Relative fold change $^{\#}$ \\
\hline DNAl1 & 3696.61 \\
GPNMB & 7.89 \\
RPGR & 83.44 \\
C7orf49 & 2.94 \\
C14orf166 & 4.92 \\
\hline & \\
DNAl1: axonemal dynein intermediate chain 1 gene; GPNMB: glycoprotein nmb \\
gene; RPGR: retinitis pigmentosa GTPase regulator gene; C7orf49: chromo- \\
some 7 open reading frame 49 gene; C14orf166: chromosome 14 open reading \\
frame 166. ${ }^{*}:$ ratio in ciliated to nonciliated cells.
\end{tabular}

Several cytoskeletal genes, such as the actin gamma 1 (ACTG1), keratin and tropomyosin genes were evidenced. Despite the fact that ciliated cells do not replicate, several cell proliferation genes were found, including the non-metastatic cells 2, protein (NM23B) expressed in gene (NME2). Among the remaining genes of table 1 , only one, the beta-tubulin gene (TUBB), had been previously demonstrated to be implicated in cilia. Finally, two genes corresponding to predicted open reading frames were identified, C7orf49 and C14orf166.

The cDNA fragments with sequences aligning to introns are reported in table 2 . These cDNA fragments are presumably representative of new isoforms of mRNA. Only the cDNA fragment mapping to the exportin 6 gene (XPO6) includes intronic and exonic sequences. The six other fragments come from intronic regions. One fragment maps to a genomic region in which two genes overlap on opposite strands (family with sequence similarity 82 (FAM82B) and copine III (CPNE3)). Since the present cloning strategy was not oriented, it is not known whether this fragment is derived from one or the other gene.

The five cDNA fragments that mapped to intergenic regions are presented in table 3 . The first two cDNA fragments (A and B) could represent a new $5^{\prime}$ or $3^{\prime}$ exon since the distance from the closest gene is at most $16 \mathrm{~kb}$. By contrast, the last two cDNA fragments (D and E) are so isolated (minimum distance of $75 \mathrm{~kb}$ from the nearest gene) that they can only be segments of new genes.

\section{Real-time PCR validation studies}

First, in order to validate the present culture system, expression of DNAI1 and RPGR, two genes which are known to be overexpressed in ciliated cells, was analysed. DNAI1 encodes a component of outer dynein arms in ciliary axonemes and is essential for ciliary function, whereas RPGR is specifically expressed in tissues containing cells with cilia or cilia-like organelles, such as retina, lung, cochlea and epithelial cells lining bronchi and sinuses [22]. For data analysis, relative quantification of target gene transcripts was performed for each sample, with normalisation to GAPDH expression, since it is an endogenous unregulated gene transcript. By real-time PCR, it was found that these two genes exhibit a 3,696- and 83fold relative increase, respectively (table 4).

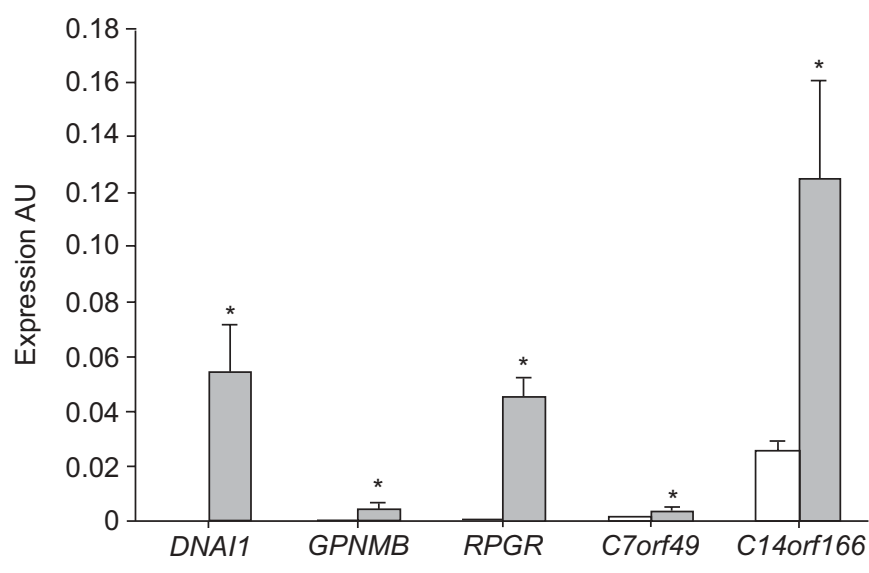

FIGURE 1. Validation of the expression data for a number of the identified human ciliated cell genes: expression in nonciliated $(\square)$, and ciliated cells ( $\square$ ). Data are presented as mean \pm SEM. AU: arbitrary unit; DNAl1: axonemal dynein intermediate chain 1 gene; GPNMB: glycoprotein $\mathrm{nmb}$ gene; RPGR: retinitis pigmentosa GTPase regulator gene; C7orf49: chromosome 7 open reading frame 49 gene; C14orf166: chromosome 14 open reading frame $166 .{ }^{*}: p<0.05$ versus nonciliated cells

Secondly, in order to validate the list of genes, the expression of one gene (GPNMB) and two open reading frames, namely C7orf49 and C14orf166, were evaluated by real-time PCR in nonciliated and ciliated cells. The three selected genes exhibited an expression in ciliated cells that was significantly higher than in nonciliated cells (fig. 1). GPNMB displayed a relative 7.89 -fold increase, a change consistent with the 3.0112.17-fold change reported by Ross et al. [12]. C7orf 49 and C14orf166 showed increased expression in human respiratory ciliated cells, with 2.94- and 4.92-fold relative changes, respectively (table 4 ).

\section{Comparison with other studies on cilia}

In order to confirm the present data, the genes list was compared to other lists obtained by various methods from the ciliated cells of various organisms (table 5). Ten out of 53 genes showing increased transcript synthesis in the present study had been reported once in other studies: annexin A1 (ANXA1); eukaryotic translation initiation factor 1 (EIF1); GPNMB; nebulette (NEBL); NME2; RPL14; secretoglobin family $1 \mathrm{~A}$ member 1 (uteroglobin) (SCGB1A1); SPATA13; thymosin beta 4, X-linked (TMSB4X); and ubiquitin A-52 residue ribosomal protein fusion product 1 (UBA52) [1, 3, 11, 12]. Two additional genes were mentioned in the studies of both OsTROWSKI et al. [1] and PAZOUR et al. [3]: ribosomal protein SA (RPSA); and ACTG1. TUBB was also detected twice [4, 8]. Finally, a computer-predicted gene, referred to as C14orf166, was mentioned three times [6-8].

In agreement with the study of PAZOUR et al. [3], it was found that FK506 binding protein 1A, 12kDa gene (FKBP1A) showed increased expression during ciliogenesis [3]. By contrast, Ross et al. [12] noted decreased expression (-2.33-fold) of this gene. Three genes that showed increased expression during ciliogenesis in the present study showed a reversed pattern in the study of Ross et al. [12]: serpin peptidase inhibitor, clade B (ovalbumin), member 2 (SERPINB2; -2.82-fold), tropomyosin 1 (TPM1; -3.31/-3.73-fold) and TPM3 (-3.49/-3.21-fold) [12]. 
TABLE 5 Genes overexpressed in human ciliated respiratory cells: genes reported in previous studies

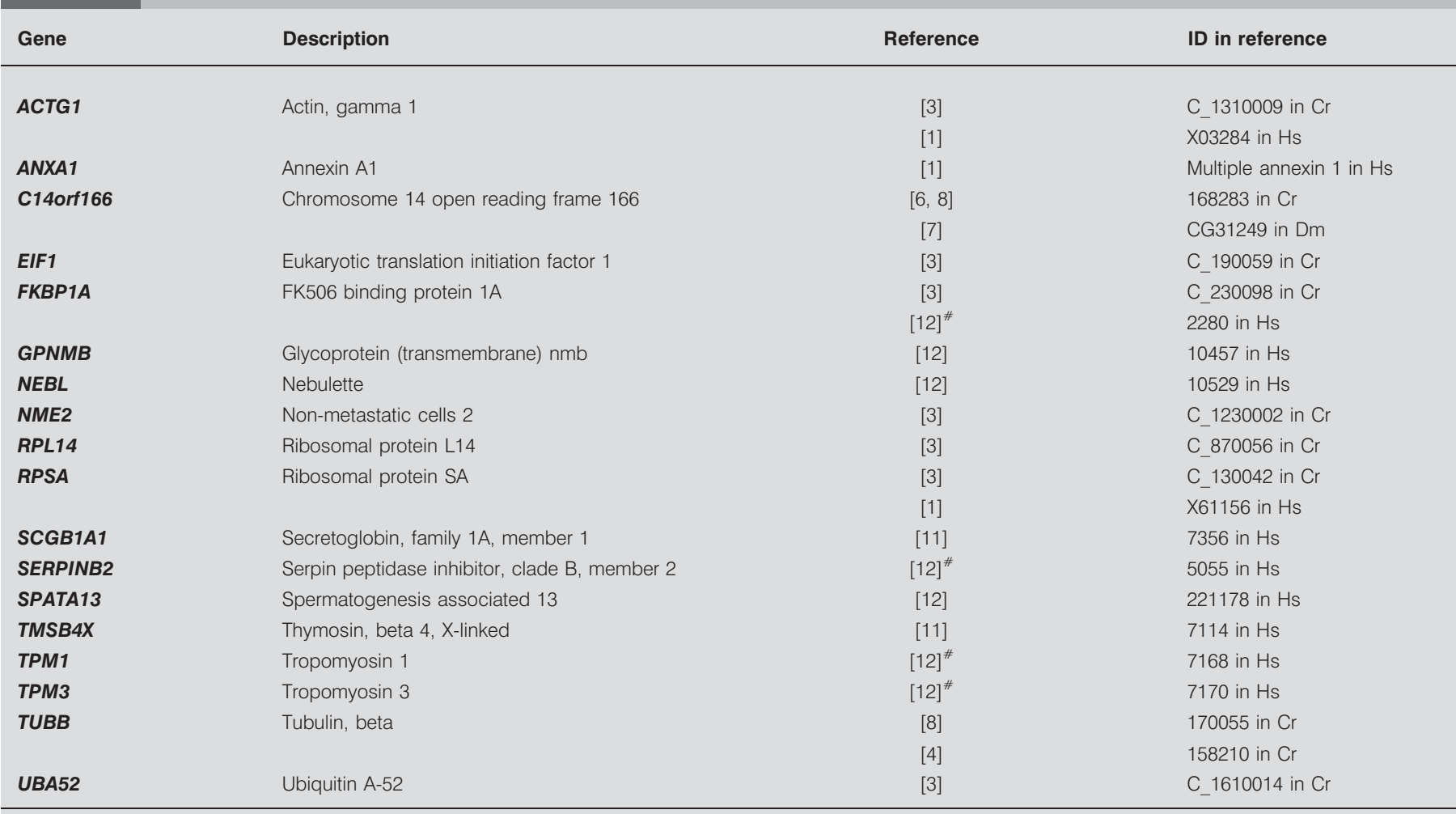

Cr: Chlamydomonas reinhardtii; Hs: Homo sapiens; Dm: Drosophila melanogaster. \#: decreased expression.

TABLE 6 Genes overexpressed in human ciliated respiratory cells: complementary DNA fragments mapping to loci related to known ciliary structure defects and diseases

\begin{tabular}{|c|c|c|c|}
\hline Defect & Location ${ }^{\#}$ & Reference & Genes at these loci \\
\hline \multicolumn{4}{|c|}{ Familial studies } \\
\hline ODA & 19q13-19qter & [24] & CXCL17" \\
\hline IDA & $15 q 13.3-15.1$ & [25] & SQRDL" \\
\hline IDA & $x$ & [26] & SLC6A14", TMSB4X \\
\hline \multicolumn{4}{|c|}{ Genomic analysis } \\
\hline PCD/SI & $3 p$ & [23] & RPL14, RPL32", RPSA \\
\hline DAD & $7 p$ & [23] & GPNMB, NDUFA4" \\
\hline PCD & 10p & [23] & DDIT4", NEBL \\
\hline SI & $11 \mathrm{q}$ & [23] & POLD4", SCGB1A1, sequence $B^{\sigma^{*+}}$ \\
\hline SI & $13 q$ & [23] & SPATA13 \\
\hline PCD & $15 q$ & [23] & TPM1 \\
\hline SI/DAD & $17 q$ & [23] & ACTG1, ATP5H \\
\hline
\end{tabular}

ODA: outer dynein arm; IDA: inner dynein arm; PCD: primary ciliary dyskinesia; SI: situs inversus; DAD: dynein arm defect; CXCL17: chemokine (CXC) ligand 17 gene; SQRDL: sulphide quinone reductase-like (yeast) gene; SLC6A14: solute carrier family 6 (amino acid transporter), member 14 gene; TMSB4X: thymosin, beta 4, X-linked gene; RPL14: ribosomal protein L14 gene; RPSA: ribosomal protein SA gene; GPNMB: glycoprotein nmb gene; NDUFA4: NADH dehydrogenase (ubiquinone) 1 alpha subcomplex, 4, 9kDa gene; DDIT4: DNA-damage-inducible transcript 4 gene; NEBL: nebulette gene; POLD4: polymerase (DNA-directed), delta 4 gene; SCGB1A1: secretoglobin, family 1A, member 1 (uteroglobin) gene; SPATA13: spermatogenesis associated 13 gene; TPM1: tropomyosin 1 gene; ACTG1: actin, gamma 1 gene; ATP5H: ATP synthase, $\mathrm{H}^{+}$ transporting, mitochondrial F0 complex, subunit d gene; CDC27: cell division cycle 27 homologue (S. cerevisiae) gene; EIF1: eukaryotic translation initiation factor 1 gene; KRT14: keratin 14 (epidermolysis bullosa simplex, Dowling-Meara, Koebner) gene; NME2: non-metastatic cells 2, protein (NM23B) expressed in gene. \#: potential loci indicated for genomic analysis; " 
The identification of causal genes in PCD and situs inversus by positional cloning is difficult because of the potentially numerous genes involved in these diseases. As a consequence, it is important to note that 22 genes from the present series map to chromosomal regions which may contain a causal gene as determined by a positional cloning approach [23-26]. These 22 genes are presented in table 6 . Half of these have never previously been reported in studies aimed at characterising components specific to cilia/flagella. Among these 22 genes, four are located in chromosomal regions showing definite linkage in familial PCD: chemokine (CXC) ligand 17 (CXCL17), sulphide quinone reductase-like (yeast) (SQRDL), solute carrier family 6 (amino acid transporter), member 14 gene and TMSB4X [24-26].

\section{DISCUSSION}

In the present study, RDA was used to identify genes differentially expressed in human ciliated respiratory cells. Subtraction of the nonciliated cell representation from that of ciliated cells resulted in the detection of 53 genes and 12 new coding sequences of known genes $(n=7)$ or putative new genes $(n=5)$. These 65 cDNA fragments are truly overexpressed in ciliated cells since a subset of three cDNAs were tested using real-time PCR analysis and all three were expressed at a significantly higher level in ciliated than nonciliated cells. In addition, 18 out of the 53 cDNAs had already been reported in other studies aimed at deciphering transcripts or proteins specific to ciliated cells. The remaining 35 cDNAs were newly identified genes overexpressed in ciliated cells. Of the sequences, 22 were located at loci related to known ciliary structure defects and diseases.

RDA is a method that is long and difficult to set up, but which is recognised as providing no false positives. Consistent with this observation, all of the cDNAs tested by real-time PCR had their overexpression confirmed, and 15 of the cDNAs of the present study had previously been reported in other studies as being overexpressed in ciliated cells. One limitation of this method is that there is selection of short cDNA fragments, meaning that there is no hope of obtaining a complete list of all overexpressed transcripts. Although microarrays provide a systematic view of transcript expression and can only detect preselected sequences, RDA has the power to detect additional expressed sequences either as novel transcript variants of known genes or even as new genes.

In table 1, a set of genes that could be linked to cells with cilia/ flagella are reported. Indeed, seven genes encoding proteins of the mitochondrial respiratory chain and oxidative phosphorylation system were identified. In ciliated cells, increased ATP production is presumably necessary for intracellular and intraflagellar transport and ciliary beating. Mutations in ACTG1, a major component of sensory ciliated cells of the cochlea, have been described as causing dominant deafness [27]. Finally, NME2 was demonstrated to be involved in spermiogenesis and flagellar movement [28], and TMSB4X was found to be highly represented in lung parenchyma and unrelated tissue types relative to the bronchial epithelium in a previous study [11].

For the real-time PCR analysis, two positive controls that have previously been described as showing increased expression in ciliated tissues, DNAI1 and RPGR, were used. Two other genes, GPNMB and C14orf166, have been reported, in others studies, to show increased expression in ciliated cells, but their function in ciliogenesis remains to be elucidated. GPNMB, a transmembrane glycoprotein, was hypothesised to be involved in growth delay and reduction of metastatic potential [29], but its role in ciliogenesis remains elusive. The C14orf166 and C7orf49 genes were predicted by bioinformatic searches of the human genome. C14orf166 is frequently detected during ciliogenesis since it has been reported by three other studies. This gene encodes protein involved in the functional regulation of human ninein in the centrosome structure $[6-8,30]$. It would be interesting to obtain complete data on C14orf166 and elucidate the biological function of C7orf49.

Finally, five totally new genes ( $\sim 10 \%$ of the whole set) were detected. Further work is warranted to characterise in detail these putative new genes, in particular sequence B, which is located in a chromosomal region implicated in situs inversus, a disturbance of lateralisation which can be secondary of ciliary dysfunction in the early embryo.

\section{ACKNOWLEDGEMENTS}

The authors warmly thank S. Picot for the contribution of the Parasitology Laboratory (University of Lyon 1, Lyon, France) to the real-time PCR experiments.

\section{REFERENCES}

1 Ostrowski LE, Blackburn K, Radde KM, et al. A proteomic analysis of human cilia: identification of novel components. Mol Cell Proteomics 2002; 1: 451-465.

2 Smith JC, Northey JG, Garg J, Pearlman RE, Siu KW. Robust method for proteome analysis by MS/MS using an entire translated genome: demonstration on the ciliome of Tetrahymena thermophila. J Proteome Res 2005; 4: 909-919.

3 Pazour GJ, Agrin N, Leszyk J, Witman GB. Proteomic analysis of a eukaryotic cilium. J Cell Biol 2005; 170: 103-113.

4 Keller LC, Romijn EP, Zamora I, Yates JR 3rd., Marshall WF., Proteomic analysis of isolated Chlamydomonas centrioles reveals orthologs of ciliary-disease genes. Curr Biol 2005; 15: 1090-1098.

5 Broadhead R, Dawe HR, Farr H, et al. Flagellar motility is required for the viability of the bloodstream trypanosome. Nature 2006; 440: 224-227.

6 Li JB, Gerdes JM, Haycraft CJ, et al. Comparative genomics identifies a flagellar and basal body proteome that includes the BBS5 human disease gene. Cell 2004; 117: 541-552.

7 Avidor-Reiss T, Maer AM, Koundakjian E, et al. Decoding cilia function: defining specialized genes required for compartmentalized cilia biogenesis. Cell 2004; 117: 527-539.

8 Stolc V, Samanta MP, Tongprasit W, Marshall WF. Genome-wide transcriptional analysis of flagellar regeneration in Chlamydomonas reinhardtii identifies orthologs of ciliary disease genes. Proc Natl Acad Sci USA 2005; 102: 3703-3707.

9 Blacque OE, Perens EA, Boroevich KA, et al. Functional genomics of the cilium, a sensory organelle. Curr Biol 2005; 15: 935-941. 
10 Swoboda P, Adler HT, Thomas JH. The RFX-type transcription factor DAF-19 regulates sensory neuron cilium formation in C. elegans. Mol Cell 2000; 5: 411-421.

11 Lonergan KM, Chari R, Deleeuw RJ, et al. Identification of novel lung genes in bronchial epithelium by serial analysis of gene expression. Am J Respir Cell Mol Biol 2006; 35: 651-661.

12 Ross AJ, Dailey LA, Brighton LE, Devlin RB. Transcriptional profiling of mucociliary differentiation in human airway epithelial cells. Am J Respir Cell Mol Biol 2007; 37: 169-185.

13 Inglis PN, Boroevich KA, Leroux MR. Piecing together a ciliome. Trends Genet 2006; 22: 491-500.

14 Lisitsyn N, Lisitsyn N, Wigler M. Cloning the differences between two complex genomes. Science 1993; 259: 946-951.

15 Hubank M, Schatz DG. Identifying differences in mRNA expression by representational difference analysis of cDNA. Nucleic Acids Res 1994; 22: 5640-5648.

16 Jorissen M, Willems T, Van der Schueren B, Verbeken E, De Boeck K. Ultrastructural expression of primary ciliary dyskinesia after ciliogenesis in culture. Acta Otorhinolaryngol Belg 2000; 54: 343-356.

17 UK Medical Research Council Laboratory of Molecular Biology, Staden Package. http://staden.sourceforge.net/ Date last updated: July 5, 2006.

18 Pearson WR, Lipman DJ. Improved tools for biological sequence comparison. Proc Natl Acad Sci USA 1988; 85: 2444-2448.

19 European Molecular Biology Laboratory-European Bioinformatics Institute, Wellcome Trust Sanger Institute, Ensembl. www.ensembl.org Date last updated: October 23, 2007. Date last accessed: December, 6: 2007.

20 National Center for Biotechnology Information, National Center for Biotechnology Information. NCBI Build 36.2. www.ncbi.nlm.nih.gov/ Date last updated: September 14, 2006. Date last accessed: December 6, 2007.
21 Pfaffl MW. A new mathematical model for relative quantification in real-time RT-PCR. Nucleic Acids Res 2001; 29: e45.

22 Iannaccone $\mathrm{A}$, Breuer DK, Wang $\mathrm{XF}$, et al. Clinical and immunohistochemical evidence for an $\mathrm{X}$ linked retinitis pigmentosa syndrome with recurrent infections and hearing loss in association with an RPGR mutation. J Med Genet 2003; 40: e118.

23 Blouin JL, Meeks M, Radhakrishna U, et al. Primary ciliary dyskinesia: a genome-wide linkage analysis reveals extensive locus heterogeneity. Eur J Hum Genet 2000; 8: 109-118.

24 Meeks M, Walne A, Spiden S, et al. A locus for primary ciliary dyskinesia maps to chromosome 19q. J Med Genet 2000; 37: 241-244.

25 Jeganathan D, Chodhari R, Meeks M, et al. Loci for primary ciliary dyskinesia map to chromosome 16p12.1-12.2 and 15q13.1-15.1 in Faroe Islands and Israeli Druze genetic isolates. J Med Genet 2004; 41: 233-240.

26 Krawczynski MR, Witt M. PCD and RP: X-linked inheritance of both disorders? Pediatr Pulmonol 2004; 38: 88-89.

27 Rendtorff ND, Zhu M, Fagerheim T, et al. A novel missense mutation in ACTG1 causes dominant deafness in a Norwegian DFNA20/26 family, but ACTG1 mutations are not frequent among families with hereditary hearing impairment. Eur J Hum Genet 2006; 14: 1097-1105.

28 Munier A, Serres C, Kann ML, et al. Nm23/NDP kinases in human male germ cells: role in spermiogenesis and sperm motility? Exp Cell Res 2003; 289: 295-306.

29 Weterman MA, Ajubi N, van Dinter IM, et al. nmb, a novel gene, is expressed in low-metastatic human melanoma cell lines and xenografts. Int J Cancer 1995; 60: 73-81.

30 Howng SL, Hsu HC, Cheng TS, et al. A novel nineininteraction protein, CGI-99, blocks ninein phosphorylation by GSK3 $\beta$ and is highly expressed in brain tumors. FEBS Lett 2004; 566: 162-168. 